PROCEEDINGS OF THE AMERICAN MATHEMATICAL SOCIETY

Volume 124, Number 8, August 1996

\title{
ON RAMANUJAN CONGRUENCES FOR MODULAR FORMS OF INTEGRAL AND HALF-INTEGRAL WEIGHTS
}

\author{
B. DATSKOVSKY AND P. GUERZHOY
}

(Communicated by Dennis A. Hejhal)

\begin{abstract}
In 1916 Ramanujan observed a remarkable congruence: $\tau(n) \equiv$ $\sigma_{11}(n) \bmod 691$. The modern point of view is to interpret the Ramanujan congruence as a congruence between the Fourier coefficients of the unique normalized cusp form of weight 12 and the Eisenstein series of the same weight modulo the numerator of the Bernoulli number $B_{12}$. In this paper we give a simple proof of the Ramanujan congruence and its generalizations to forms of higher integral and half-integral weights.
\end{abstract}

\section{INTRODUCTION}

Let $\tau(n)$ be given by $q \prod_{n=1}^{\infty}\left(1-q^{n}\right)^{24}=\sum_{n=1}^{\infty} \tau(n) q^{n}$, and let $\sigma_{11}(n)=$ $\sum_{d \mid n} d^{11}$. Ramanujan [10] observed that

$$
\tau(n) \equiv \sigma_{11}(n) \quad \bmod 691 .
$$

The modern point of view is to interpret the Ramanujan congruence as a congruence between the Fourier coefficients of the unique normalized cusp form

$$
\Delta(z)=q \prod_{n=1}^{\infty}\left(1-q^{n}\right)^{24}, \quad q=e^{2 \pi i z},
$$

of weight 12 and the Eisenstein series

$$
G_{12}(z)=\frac{691}{65520}+\sum_{n=1}^{\infty} \sigma_{11}(n) q^{n}, \quad q=e^{2 \pi i z},
$$

of the same weight. In the last twenty years this point of view has inspired a number of very deep investigations, such as those of Katz [4], Manin [9], Ribet [11]-[13], Serre [14], [15], and Swinnerton-Dyer [17], [18], concerning congruences for the Fourier coefficients of modular forms. In this paper we give a simple proof of the Ramanujan congruence and its generalizations to forms of higher weights. Although discovered independently, our argument is very similar to the one used in [12] for slightly different purposes. However, since this argument seems not to be generally well known, we dare to present it to the public here.

Received by the editors May 15, 1994.

1991 Mathematics Subject Classification. Primary 11F33; Secondary 11F30, 11F32, 11F37.

The first author's research was supported by a Fulbright fellowship.

(C)1996 American Mathematical Society 
The Fourier coefficients of modular forms of half-integral weight (see, for example, [1]) are very interesting arithmetic objects. In view of the Shimura correspondence between modular forms of weight $k+\frac{1}{2}$ and modular forms of weight $2 k$, it is natural to ask which of the congruences for the Fourier coefficients of modular forms of weight $2 k$ carry over to a congruence for the Fourier coefficients of modular forms of weight $k+\frac{1}{2}$. In [8] Maeda found an example in which a congruence modulo $p$ between two cusp forms of integral weight descends via the Shimura correspondence to a congruence modulo $p$ between cusp forms of half-integral weight, and in [6] Koblitz observed that the Ramanujan congruence modulo 691 carries over to a congruence modulo 691 for modular forms of weight $13 / 2$. In this paper we show that under some additional conditions Ramanujan congruences for modular forms of weight $2 k$ carry over to congruences for modular forms of weight $k+\frac{1}{2}$.

\section{RAMANujan CONGRUENCES FOR FORMS OF INTEGRAL WEIGHTS}

Let $K$ be an algebraic number field with the ring of algebraic integers $O_{K}$, and let $\mathcal{I}$ be an ideal of $O_{K}$. Let $O_{\mathcal{I}}$ denote the ring $S_{\mathcal{I}}^{-1} O_{K}$ where $S_{\mathcal{I}}=\left\{a \in O_{K}\right.$ : $(a, \mathcal{I})=1\}$.

Definition. We call two modular forms $f$ and $g$ on $S L(2, \mathbf{Z})$ congruent modulo $\mathcal{I}$, and write $f \equiv g \bmod \mathcal{I}$, if all Fourier coefficients of $f-g$ are in $\mathcal{I} O_{\mathcal{I}}$.

Note that in the definition above we do not require, as is usually done, that the coefficients of $f$ and $g$ be in $O_{\mathcal{I}}$. The reason for this is that in the next section we will establish congruences for the Cohen series $\mathcal{H}_{k}(z)$ (see Theorems 3 and 4 below) modulo certain prime ideals $\mathcal{P}$. The Fourier coefficients of $\mathcal{H}_{k}(z)$ are rational numbers, and we cannot be sure that their denominators are relatively prime to $\mathcal{P}$. Our definition allows us to ignore this problem.

Let

$$
G_{k}(z)=-\frac{B_{k}}{2 k}+\sum_{n=1}^{\infty} \sigma_{k-1}(n) q^{n}, \quad q=e^{2 \pi i z},
$$

where $B_{k}$ is the $k^{\text {th }}$ Bernoulli number, be the normalized Eisenstein series of weight $k$, and let $N_{k}$ denote the numerator of the reduced fraction $\frac{B_{k}}{2 k}$. Then we have

Theorem 1. For any even integer $k \geq 12$ there exists a nonzero cusp form $f$ of weight $k$ with rational Fourier coefficients such that

$$
f \equiv G_{k} \quad \bmod N_{k} .
$$

Proof. Let $E_{k}=-\frac{2 k}{B_{k}} G_{k}$ be the Eisenstein series of weight $k$, normalized so that its constant term is equal to 1 . It is well known (see, for instance, [5], p.111) that

$$
E_{4}(z)=1+240 \sum_{n=1}^{\infty} \sigma_{3}(n) q^{n}, \quad q=e^{2 \pi i z},
$$

and

$$
E_{6}(z)=1-504 \sum_{n=1}^{\infty} \sigma_{5}(n) q^{n}, \quad q=e^{2 \pi i z} .
$$

Note that $E_{4}$ and $E_{6}$ have integral Fourier coefficients. Using $E_{4}$ and $E_{6}$ it is now easy to construct a form $h$ of weight $k$ with the constant term 1 and integral Fourier coefficients. Let $f=G_{k}+\frac{B_{k}}{2 k} h$. Then $f$ is a cusp form with rational 
Fourier coefficients; moreover, $f \equiv G_{k} \bmod N_{k}$. Since $N_{k}>1$ for $k \geq 12$ and the first Fourier coefficient of $G_{k}$ is $1, f \neq 0$.

Let $S_{k}$ denote the space of cusp forms of weight $k$. When $\operatorname{dim} S_{k}=1$ we have the following corollary:

Corollary 1.1. Suppose $\operatorname{dim} S_{k}=1$. Let $f_{k}$ denote the unique cusp form of weight $k$ normalized so that its first Fourier coefficient is 1 . Then

$$
f_{k} \equiv G_{k} \quad \bmod N_{k} .
$$

Proof. By Theorem $1 G_{k}$ is congruent to some multiple of $f_{k}$ modulo $N_{k}$. Since the first Fourier coefficients of $G_{k}$ and $f_{k}$ are both $1, G_{k} \equiv f_{k} \bmod N_{k}$.

When $k=12$, Corollary 1.1 yields the Ramanujan congruence modulo 691. For $k=16,18,20,22$, and 26 , it implies the following congruences, first obtained by Manin [9]:

$$
\begin{gathered}
\sigma_{15}(n) \equiv \tau(n)+240 \sum_{m=1}^{n-1} \sigma_{3}(m) \tau(n-m) \bmod 3617 \\
\sigma_{17}(n) \equiv \tau(n)-504 \sum_{m=1}^{n-1} \sigma_{5}(m) \tau(n-m) \bmod 43867 ; \\
\sigma_{19}(n) \equiv \tau(n)+480 \sum_{m=1}^{n-1} \sigma_{7}(m) \tau(n-m) \bmod 283 \cdot 617 ; \\
\sigma_{21}(n) \equiv \tau(n)-264 \sum_{m=1}^{n-1} \sigma_{9}(m) \tau(n-m) \bmod 131 \cdot 593 ; \\
\sigma_{25}(n) \equiv \tau(n)-24 \sum_{m=1}^{n-1} \sigma_{13}(m) \tau(n-m) \bmod 657931 .
\end{gathered}
$$

Now let $\operatorname{dim} S_{k} \geq 2$. We call a cusp form primitive if it is a common eigenfunction of all Hecke operators, normalized so that its first Fourier coefficient is equal to 1. The coefficients of primitive forms are algebraic integers in some algebraic number field $K$. The following theorem is a higher weight analogue of Corollary 1.1:

Theorem 2. Let $t=\operatorname{dim} S_{k}$, and let $\phi_{1}, \ldots, \phi_{t}$ be the primitive Hecke eigenforms in $S_{k}$. Let $K$ be an algebraic number field containing the Fourier coefficients of $\phi_{1}, \ldots, \phi_{t}$, and let $\mathcal{P}$ be a prime ideal of $O_{K}$ that divides $N_{k}$. Then there exists a primitive Hecke eigenform $\phi_{i}$ in $S_{k}$ such that

$$
\phi_{i} \equiv G_{k} \quad \bmod \mathcal{P} .
$$

This theorem follows easily from Theorem 1 and Lemma 6.11 of [2]. However, in order to make this paper self-contained we present the proofs of this theorem and an appropriate lemma in their entirety below. 
Lemma 2.1. Suppose that for some integer $m$ there exist algebraic integers $\beta_{0}, \ldots$, $\beta_{t} \in K, \beta_{0} \not \equiv 0 \bmod \mathcal{P}^{m}$, such that

$$
\sum_{i=1}^{t} \beta_{i} \phi_{i} \equiv \beta_{0} G_{k} \quad \bmod \mathcal{P}^{m}
$$

Then, for some $i, \phi_{i} \equiv G_{k} \bmod \mathcal{P}$.

Proof. Pick a minimal subset of $\left\{\phi_{1}, \ldots, \phi_{t}\right\}$, say $\left\{\phi_{1}, \ldots, \phi_{\ell}\right\}$, such that for some $m$ there exist integers $\beta_{0}, \ldots, \beta_{\ell} \in K, \beta_{0} \not \equiv 0 \bmod \mathcal{P}^{m}$, for which

$$
\sum_{i=1}^{\ell} \beta_{i} \phi_{i} \equiv \beta_{0} G_{k} \quad \bmod \mathcal{P}^{m}
$$

Applying the operator $T_{n}$ to (1) and eliminating the eigenform $\phi_{1}$, we obtain

$$
\sum_{i=2}^{\ell} \beta_{i}\left(\alpha_{i}(n)-\alpha_{1}(n)\right) \phi_{i} \equiv \beta_{0}\left(\sigma_{k-1}(n)-\alpha_{1}(n)\right) G_{k} \quad \bmod \mathcal{P}^{m} .
$$

This contradicts minimality of the set $\left\{\phi_{1}, \ldots, \phi_{\ell}\right\}$ unless $\sigma_{k-1}(n) \equiv \alpha_{1}(n) \bmod$ $\mathcal{P}$. Hence, for every $n, \sigma_{k-1}(n) \equiv \alpha_{1}(n) \bmod \mathcal{P}$. But $\alpha_{1}(n)$ is the $n^{\text {th }}$ Fourier coefficient of the primitive Hecke eigenform $\phi_{1}$. Hence $\phi_{1} \equiv G_{k} \bmod \mathcal{P}$.

Proof of Theorem 2. By Theorem 1 there exists a nonzero form $f$ in $M_{k}$ with rational Fourier coefficients such that $f \equiv G_{k} \bmod N_{k}$. Write $f=\sum_{i=1}^{t} \gamma_{i} \phi_{i}$ where $\gamma_{i} \in K$. Let $\gamma \in K$ be an algebraic integer such that, for every $i, \beta_{i}=\gamma \gamma_{i}$ is an algebraic integer. Then $\sum_{i=1}^{t} \beta_{i} \phi_{i} \equiv \gamma G_{k} \bmod \gamma N_{k}$. Let $\operatorname{ord}_{\mathcal{P}} \gamma=m-1$. Since $\mathcal{P} \mid N_{k}$, the last congruence can be rewritten as $\sum_{i=1}^{t} \beta_{i} \phi_{i} \equiv \gamma G_{k} \bmod \mathcal{P}^{m}$. Moreover, $\gamma \not \equiv 0 \bmod \mathcal{P}^{m}$. By Lemma 2.1 there exists a primitive Hecke eigenform $\phi_{i}$ that is congruent to $G_{k}$ modulo $\mathcal{P}$.

When $\operatorname{dim} S_{k}$ is low, and in particular when $\operatorname{dim} S_{k}=2$, Theorem 2 has an "elementary" arithmetic flavor. As an illustration of this flavor we are now going to consider in more detail the weight $k=24$. In this case $\operatorname{dim} S_{24}=2$, and $S_{24}$ is generated over the field of complex numbers $\mathbf{C}$ by two cusp forms $\Delta^{2}$ and $\Delta G_{12}$. Calculations ${ }^{1}$ show that $K=\mathbf{Q}(\sqrt{144169})$ and the two primitive forms are

$$
\begin{aligned}
& u=\left(\frac{324204}{691}-12 \sqrt{144169}\right) \Delta^{2}+\frac{65520}{691} \Delta G_{12} \\
& v=\left(\frac{324204}{691}+12 \sqrt{144169}\right) \Delta^{2}+\frac{65520}{691} \Delta G_{12} .
\end{aligned}
$$

The prime divisors of the numerator of $B_{24} / 48$ are 103 and 2294797. They both split in $K$ :

$$
\begin{gathered}
103=(103,22+\sqrt{144169})(103,22-\sqrt{144169}) \\
2294797=(2294797,1022092+\sqrt{144169})(2294797,1022092-\sqrt{144169}) .
\end{gathered}
$$

One can check that in accordance with Theorem 2

$$
u \equiv G_{24} \quad \bmod (2294797,1022092-\sqrt{144169})(103,22+\sqrt{144169})
$$

\footnotetext{
${ }^{1}$ All specific calculations were carried out on a computer using Mathematica.
} 
and

$$
v \equiv G_{24} \quad \bmod (2294797,1022092+\sqrt{144169})(103,22-\sqrt{144169}) .
$$

One can also rewrite these congruences in elementary way, i.e. using only arithmetic functions $\tau(n)$ and $\sigma_{k}(n)$ :

$$
\sum_{\substack{n=a+b \\ a, b \geq 0}} \tau(a)\left(\tau(b)\left(\frac{324204}{691}+264\right)+\frac{65520}{691} \sigma_{11}(b)\right) \equiv \sigma_{23}(n) \quad \bmod 103,
$$

and

$$
\sum_{\substack{n=a+b \\ a, b \geq 0}} \tau(a)\left(\tau(b)\left(\frac{324204}{691}-791119\right)+\frac{65520}{691} \sigma_{11}(b)\right) \equiv \sigma_{23}(n) \quad \bmod 2294797 .
$$

Here $\sigma_{k}(0)$ stands for the number $-B_{k} / 2 k$.

\section{RAMANUJAN CONGRUENCES FOR FORMS OF HALF-INTEGRAL WEIGHT}

The number of ingredients that enter in the proof of Theorem 1 is surprisingly small. All we need are the following facts:

1) The space of cusp forms has codimension 1 in the space of modular forms.

2) There exists a modular form with integral Fourier coefficients and the constant term equal to 1 .

Let $M_{k+\frac{1}{2}}^{+}(4)$ be the subspace of the space of modular forms of weight $k+\frac{1}{2}$ on $\Gamma_{0}(4)$ consisting of those modular forms whose $n^{\text {th }}$ Fourier coefficients vanish whenever $(-1)^{k} n \equiv 2,3 \bmod 4$, and let $S_{k+\frac{1}{2}}^{+}(4)$ denote the space of cusp forms in $M_{k+\frac{1}{2}}^{+}(4)$. Then by [7], Proposition 1 ,

$$
M_{k+\frac{1}{2}}^{+}(4)=\mathbf{C} \mathcal{H}_{k} \oplus S_{k+\frac{1}{2}}^{+}(4),
$$

where

$$
\mathcal{H}_{k}(z)=\sum_{n=0}^{\infty} H(k, n) q^{n}, \quad q=e^{2 \pi i z}
$$

is the Eisenstein series of Cohen [1]. We note that $H(k, 0)=\zeta(1-2 k)=-\frac{B_{2 k}}{2 k}$ and for $n$ square-free $H(k, n)=L\left(1-k, \chi_{(-1)^{k} n}\right)$, where $\chi_{D}$ is the real quadratic character $\chi_{D}(d)=\left(\frac{D}{d}\right)$.

By Proposition 1 of [7] $M_{k+\frac{1}{2}}^{+}(4) \cong M_{k} \oplus M_{k-2}$ if $k$ is even, and $M_{k+\frac{1}{2}}^{+}(4) \cong$ $M_{k-3} \oplus M_{k-5}$ if $k$ is odd. The isomorphism from $M_{k} \oplus M_{k-2}, k$ even, (resp. $M_{k-3} \oplus M_{k-5}, k$ odd) to $M_{k+\frac{1}{2}}^{+}(4)$ is effected as follows:

$$
\begin{gathered}
(g(z), h(z)) \rightarrow g(4 z) \theta(z)+h(4 z) H_{2}(z) \\
\left(\operatorname{resp} .(g(z), h(z)) \rightarrow g(4 z) H_{3}(z)+h(4 z) H_{5}(z)\right),
\end{gathered}
$$

where $\theta(z)=\sum_{n \in \mathbf{Z}} q^{n^{2}}, q=e^{2 \pi i z}$, and $H_{k}(z)=-\frac{k}{B_{k}} \mathcal{H}_{k}(z)$ is the Cohen series, normalized so that its constant term is equal to 1 . It is easy to check (see [1], 
Corollary 3.2) that $\theta(z), H_{2}, H_{3}$ and $H_{5}$ all have integral Fourier coefficients. It follows that for any $k$ there exists a form $h(z) \in M_{k+\frac{1}{2}}^{+}(4)$ with integral Fourier coefficients and the constant term equal to 1 . Thus the conditions 1) and 2) above are satisfied, and we have:

Theorem 3. Let $N_{2 k}^{\prime}$ denote the numerator of the reduced fraction $\frac{B_{2 k}}{2 k}$. Then there exists a cusp form $f \in S_{k+\frac{1}{2}}^{+}(4)$ with rational Fourier coefficients such that

$$
\mathcal{H}_{k} \equiv f \quad \bmod N_{2 k}^{\prime} \text {. }
$$

The following corollary was first observed by Koblitz [6] in the case when $k=6$.

Corollary 3.1. Suppose $\operatorname{dim} S_{k+\frac{1}{2}}^{+}(4)=1$. Let $f_{k}$ denote the unique cusp form in $S_{k+\frac{1}{2}}^{+}(4)$ whose first non-zero Fourier coefficient equals to 1 . Then

$$
\mathcal{H}_{k} \equiv\left\{\begin{array}{ll}
H(k, 1) f_{k} & \text { if } k \text { is even } \\
H(k, 3) f_{k} & \text { if } k \text { is odd }
\end{array} \quad \bmod N_{2 k}^{\prime} .\right.
$$

Proof. Using Proposition 1 of [7] and Corollary 3.2 of [1], one can show that $S_{k+\frac{1}{2}}^{+}(4)$ contains a cusp form with integral Fourier coefficients and the first non-zero coefficient equal to 12 if $k$ is even and to 144 if $k$ is odd. Since for any $k$ such that $\operatorname{dim} S_{k+\frac{1}{2}}^{+}(4)=1$ we have $\left(N_{2 k}^{\prime}, 12\right)=1$, it follows that $f_{k}$ has $N_{2 k}^{\prime}$-integral Fourier coefficients. By Theorem $3, \mathcal{H}_{k}$ is congruent to a constant multiple of $f_{k}$ modulo $N_{2 k}^{\prime}$. Comparing the first non-zero Fourier coefficient of this multiple with the corresponding coefficient of $\mathcal{H}_{k}$, we get the corollary above.

Let $p$ be a prime. If $f=\sum_{n=0}^{\infty} a_{n} q^{n}, q=e^{2 \pi i z}$, is in $M_{k+\frac{1}{2}}^{+}(4)$, we can define the Hecke operator $T_{k+\frac{1}{2}}^{+}\left(p^{2}\right)$ by

$$
f \mid T_{k+\frac{1}{2}}^{+}\left(p^{2}\right)=\sum_{n=0}^{\infty}\left(a\left(p^{2} n\right)+\left(\frac{(-1)^{k} n}{p}\right) p^{k-1} a(n)+p^{2 k-1} a\left(\frac{n}{p^{2}}\right)\right) q^{n}
$$

where we put $a(x)=0$ if $x$ is not an integer. The operators $T_{k+\frac{1}{2}}^{+}\left(p^{2}\right)$ form an algebra which is isomorphic to the Hecke algebra of $M_{2 k}$ (this isomorphism sends $T_{k+\frac{1}{2}}^{+}\left(p^{2}\right)$ to $\left.T_{2 k}(p)\right)$; moreover, by Theorem 1 of [7] $M_{k+\frac{1}{2}}^{+}(4)$ and $M_{2 k}$ are isomorphic as Hecke algebra modules.

More generally, one can define Hecke operators $T_{k+\frac{1}{2}}\left(p^{2}\right)$ on the space $M_{k+\frac{1}{2}}\left(\Gamma_{0}(N)\right)$ of modular forms of weight $k+\frac{1}{2}$ and level $N$ where $4 \mid N$. These operators are Hermitian and self-adjoint with respect to the Petersson inner product, and commute with each other. Therefore the space $S_{k+\frac{1}{2}}\left(\Gamma_{0}(N)\right)$ of cusp forms of weight $k+\frac{1}{2}$ and level $N$ has a basis of Hecke eigenforms.

Let $\psi \in S_{k+\frac{1}{2}}\left(\Gamma_{0}(N)\right)$ be a Hecke eigenform for the operators $T_{k+\frac{1}{2}}\left(p^{2}\right)$ with the corresponding eigenvalues $\lambda_{p}$. Let $b(n)$ be given by the formal identity

$$
\sum_{n=1}^{\infty} b_{n} n^{-s}=\prod_{p}\left(1-\lambda_{p} p^{-s}+p^{2 k-1-2 s}\right)^{-1}
$$


Let $\phi(z)=\sum_{n=1}^{\infty} b(n) q^{n}, q=e^{2 \pi i z}$. Shimura [16] showed that $\phi(z) \in M_{2 k}\left(\Gamma_{0}\left(N^{\prime}\right)\right)$ for some integer $N^{\prime}$ and that if $k \geq 2$ then $\phi$ is a cusp form. In fact, the identity (3) shows that $\phi$ is a Hecke eigenform of weight $2 k$ and that $\phi \mid T_{2 k}(p)=\lambda_{p} \phi$. The map that sends $\psi$ to $\phi$ is called the Shimura correspondence or the Shimura lifting.

We will now attempt to show that the Ramanujan congruences descend via the Shimura correspondence from forms of integral to forms of half-integral weights. Since in half-integral weight a congruence for the eigenvalues of the Hecke operators no longer implies a congruence for the Fourier coefficients of the corresponding Hecke eigenform, Lemma 2.1 is no longer valid. However, a weaker version of Theorem 2 survives:

Theorem 4. Let $K$ be a field containing all the eigenvalues of the Hecke operators on $M_{2 k}$, and let $\mathcal{P}$ be a prime ideal in $O_{K}$ that divides $N_{2 k}$. Suppose that $\mathcal{H}_{k} \not \equiv$ $0 \bmod \mathcal{P}$. Then there exist Hecke eigenforms $\psi_{i} \in S_{k+\frac{1}{2}}^{+}(4)$ such that

$$
\mathcal{H}_{k} \equiv \sum \psi_{i} \quad \bmod \mathcal{P}
$$

and the primitive forms $\phi_{i} \in S_{2 k}$ corresponding to $\psi_{i}$ under the Shimura correspondence satisfy $\phi_{i} \equiv G_{2 k} \bmod \mathcal{P}$.

Corollary 4.1. Suppose a primitive form $\phi_{i} \in S_{2 k}$ satisfying $\phi_{i} \equiv G_{2 k} \bmod \mathcal{P}$ is unique. Suppose further that $\mathcal{H}_{k} \not \equiv 0 \bmod \mathcal{P}$. Then there exists a common Hecke eigenform $\psi_{i} \in S_{k+\frac{1}{2}}^{+}(4)$ corresponding to $\phi_{i}$ under the Shimura correspondence such that

$$
\mathcal{H}_{k} \equiv \psi_{i} \bmod \mathcal{P} \text {. }
$$

Proof of Theorem 4. Write the form $f$ of Theorem 3 as a sum of Hecke eigenforms: $f=\sum_{i=1}^{t} \varphi_{i}$. The $\varphi_{i}$ correspond to $\phi_{i} \in S_{2 k}$ under the Shimura correspondence, and we may assume that $\phi_{i} \equiv G_{2 k} \bmod \mathcal{P}, i=1, \ldots, \ell$, and $\phi_{i} \not \equiv G_{2 k} \bmod \mathcal{P}$ if $i>\ell$. Then for any $i>\ell$ there exists a Hecke operator $T\left(n_{i}\right)$ such that the eigenvalue $\alpha_{i}\left(n_{i}\right) \not \equiv \sigma_{2 k-1}\left(n_{i}\right) \bmod \mathcal{P}$. By Theorem 3

$$
\mathcal{H}_{k} \equiv \sum_{i=1}^{t} \varphi_{i} \quad \bmod \mathcal{P}
$$

Applying the operators $T_{k+\frac{1}{2}}^{+}\left(n_{i}^{2}\right)$ and eliminating the eigenforms $\varphi_{i}, i>\ell$, we obtain

$$
\prod_{i=\ell+1}^{t}\left(\sigma_{2 k-1}\left(n_{i}\right)-\alpha_{i}\left(n_{i}\right)\right) \mathcal{H}_{k} \equiv \sum_{j=1}^{\ell} \prod_{i=\ell+1}^{t}\left(\alpha_{j}\left(n_{i}\right)-\alpha_{i}\left(n_{i}\right)\right) \varphi_{j} \quad \bmod \mathcal{P} .
$$

Note that because $\mathcal{H}_{k} \not \equiv 0 \bmod \mathcal{P}$ the sum on the right hand side of (4) is not empty. Since $\prod_{i=\ell+1}^{t}\left(\sigma_{2 k-1}\left(n_{i}\right)-\alpha_{i}\left(n_{i}\right)\right) \not \equiv 0 \bmod \mathcal{P}$, we can divide by it to obtain our theorem.

We do not know how to prove the uniqueness hypothesis under which Corollary 4.1 holds; nor do we have an argument that shows that $\mathcal{H}_{k} \not \equiv 0 \bmod \mathcal{P}$. The latter is clearly the case if $B_{2 k} / 2 k$ is relatively prime to $H(k, 1)=\zeta(1-k)$ if $k$ is even and to $H(k, 3)=L\left(1-k, \chi_{-3}\right)$ if $k$ is odd. A quick look at the tables (see [19]) 
shows that for $k \leq 60 B_{2 k} / 2 k$ is relatively prime to $B_{k} / k$. Moreover, $\left|B_{2 k} / 2 k\right|$ is asymptotic to $\frac{2(2 k-1) !}{(2 \pi)^{2 k}}$ whereas $|H(k, 1)|$ is asymptotic to $\frac{2(k-1) !}{(2 \pi)^{k}}$ and $|H(k, 3)|$ is

asymptotic to $2 \frac{3^{k-\frac{1}{2}}(k-1) !}{(2 \pi)^{k}}$. This suggests that large prime divisors of $B_{2 k} / 2 k$ do not divide $H(k, 1)$ or $H(k, 3)$. Of course, it may happen that $B_{2 k} / 2 k$ has only small prime divisors.

As for the uniqueness hypothesis, it is clearly valid when $\operatorname{dim} S_{2 k}=1$, and an easy computer calculation shows that it holds for any $\mathcal{P}$ dividing $N_{2 k}$ when $\operatorname{dim} S_{2 k}=2$. Several heuristic arguments showing why we should expect uniqueness to hold fairly often can be found in [3]. Thus Ramanujan congruences often carry through the Shimura correspondence from forms of integral weight on $S L(2, \mathbf{Z})$ to forms of half-integral weight on $\Gamma_{0}(4)$.

\section{ACKNOWLEDGEMENTS}

The authors are grateful to Prof. K. Ribet for a useful communication. This paper was written while the first author was a guest at the Technion, Israel Institute of Technology, supported by a Fulbright fellowship. He wishes to thank Technion for its hospitality and the US-Israel Education Foundation for its generous support.

\section{REFERENCES}

[1] Cohen, H., Sums involving the values at negative integers of L-functions of quadratic characters, Math. Ann. 217 (1975), 271-285. MR 52:3080

[2] Deligne, P. and Serre, J.-P., Formes modulaires de poids 1, Ann. Sci. Ecole Norm. Sup. 7 (1974), 507-530. MR 52:284

[3] Guerzhoy, P., On Ramanujan congruences between special values of Hecke and Dirichlet $L$-functions, preprint.

[4] Katz, N. M., Higher congruences between modular forms, Ann. Math. 101 (1975), 332-367. MR 54:5120

[5] Koblitz, N., Introduction to Elliptic Curves and Modular Forms, Springer-Verlag, New YorkHeidelberg-Berlin, 1984. MR 86c: 11040

[6] Koblitz, N., p-Adic congruences and modular forms of half integer weight, Math. Ann. 274 (1986), 199-220. MR 88a:11043

[7] Kohnen, W., Modular forms of half-integral weight on $\Gamma_{0}(4)$, Math. Ann. 248 (1980), 249266. MR 81j: 10030

[8] Maeda, Y., A congruence between modular forms of half-integral weight, Hokkaido Math. J. 12 (1983), 64-73. MR 84e:10036

[9] Manin, Y. I., Periods of parabolic forms and p-adic Hecke series, Math. USSR Sbornik 92 (1973), 378-401; English transl., Math. USSR Sbornik 21 (1973), 371-393. MR 49:10638

[10] Ramanujan, S., On certain arithmetic functions, Trans. Cambridge Phil. Soc. 22 (1916), 159-184.

[11] Ribet, K. A., On l-adic representations attached to modular forms, Invent. Math. 28 (1975), 245-275. MR 54:7379

[12] Ribet, K.A., A modular construction of unramified p-extensions of $Q\left(\mu_{p}\right)$, Invent. Math. 34 (1976), 151-162. MR 54:7424

[13] Ribet, K. A., Congruence relations between modular forms, Proceedings of the International Congress of Mathematicians (Warsaw, 1983), PWN, Warsaw, 1984, pp. 503-514. MR 87c: 11045

[14] Serre, J.-P., Congruences et formes modulaires (d'après H. P. F. Swinnerton-Dyer), Lect. Notes Math. 317, Springer-Verlag, Berlin-Heidelberg-New York, 1973, pp. (319-338). MR $57: 5904 a$

[15] Serre, J.-P., Divisibilité des coefficients des formes modulaires de poids entiers, C. R. Acad. Sci. Paris 279 (1974), 679-682. MR 52:3060

[16] Shimura, G., On modular forms of half integral weight, Ann. Math. 97 (1973), 440-481. MR 48:10989 
[17] Swinnerton - Dyer, H. P. F., On l-adic representations and congruences for coefficients of forms, Lect. Notes Math. 350, Springer-Verlag, Berlin-Heidelberg-New York, 1973, pp. 1- 55. MR 53:10717

[18] Swinnerton - Dyer, H. P. F., Congruence properties of $\tau(n)$, in Ramanujan Revisited, Proceedings of the Centenary Conference, Academic Press, San Diego-London, 1988. MR 89e:10028

[19] Wagstaff, S., The irregular primes to 125,000, Math. Comp. 32 (1978), 583-591. MR 58:10711

Department of Mathematics, Temple University, Philadelphia, Pennsylvania 19122

E-mail address: bdats@euclid.math.temple.edu

Department of Mathematics, Technion-Israel Institute of Technology, 32000 Haifa, ISRAEL

E-mail address: pasha@techunix.technion.ac.il

Current address: Fakultät für Mathematik und Informatik, Universität Mannheim, D-6800 Mannheim 1, Germany

E-mail address: pasha@euklid.math.uni-mannheim.de 